\title{
Oxford Handbook of Anaesthesia, Fourth Edition
}

\author{
Keith G. Allman, Iain H. Wilson (Editors). Oxford University Press, Oxford, 2016. \\ Price: \$57.50. Pages: 1266. ISBN 978-0-19-871941-0
}

\author{
Sophie N. Davie, MD
}

Received: 5 May 2016/Revised: 19 May 2016/Accepted: 1 June 2016/Published online: 6 June 2016

(c) Canadian Anesthesiologists' Society 2016

Anesthesiology is a vast, complex field. Thus, the knowledge base required to become a safe, competent anesthesiologist is immense. Medical students and junior residents often find it difficult to broach the subject. In part, this book provides the reader with a quick overview of the multitude of topics faced by the anesthesiologist. Reading this book cover to cover, for example, would provide the student interested in anesthesiology with a good understanding of the scope of the field as well as its core concepts. It also serves as a quick reference for information that even the more seasoned practitioner may require on less frequent occasions.

The book is well organized into logical topic headings and subheadings. The body of the text is in bullet-point form and lends itself to quick review of the anesthetic considerations for disease states and surgical procedures, as well as subspecialty-specific topics.

The handbook is divided into three broad sections. The first section focuses on physiology and pathophysiology, with a chapter dedicated to each organ system. These chapters consist of a review of each disease within its organ system, thus providing information on how that disease is relevant to the anesthesiologist. The summaries of each disease are succinct and highlight relevant points, including a brief description of the pathophysiology along with general considerations and key features of the preoperative assessment relevant to that disease. This information is followed by a review of pertinent investigations and preoperative optimization, if relevant, as well as the

S. N. Davie, MD ( ()

Department of Anesthesia and Perioperative Medicine,

University of Manitoba, Winnipeg, MB, Canada

e-mail: umdavie@myumanitoba.ca suggested conduct of anesthesia and postoperative care. Importantly, the majority of topics in this section are supplemented with a "further reading" list, where the reader is referred to a more comprehensive review of the topic. These chapters would be particularly useful for an anesthesia student first approaching the disease in question or for any learner who needs a high-yield, concise summary of any of the major diseases relevant to anesthesiology.

The second section of the book is dedicated to the anesthetic considerations of specific operations, with each chapter focusing on a different surgical subspecialty. Each of the chapters in this section begins with a discussion of the general principles necessary to understand the anesthetic issues relevant to that surgical specialty. For example, the chapter on neurosurgery begins with a review of intracranial pressure, including the effects of several anesthetic agents, and the management of increased intracranial pressure. The chapters in this section contain one- or two-page summaries of each of the common operations performed within each surgical subspecialty. Each summary consists of a table with a brief description of the surgery, the typical time required, the amount of pain associated with the surgery, patient positioning, expected blood loss, and practical techniques, including whether an endotracheal tube, arterial line, or central venous line is required. The remainder of the summary is divided into preoperative, perioperative, and postoperative considerations for each operation, as well as any special considerations. This section is a great resource for any medical student or resident requiring a brief review on a specific surgical intervention because all of the key concepts required to deliver an anesthetic safely are covered in a concise, easy-to-understand manner. 
The book's third section is dedicated to the various anesthetic subspecialties, such as obstetric and pediatric anesthesia, acute pain, the critically ill, and regional anesthesia. There are also chapters focusing on other key areas of importance to anesthesiologists, such as anesthetic emergencies, airway assessment, management of blood products, and postoperative nausea. Consistent with the other sections in this book, this section is easy to read and well organized. The chapter on consent and anesthetic risk is a useful reference for all learners at any stage of their training. The chapter contains several tables that aid in the communication of risk to patients in a straightforward manner. I found the table delineating the incidence of a wide variety of anesthetic complications relating to both general and regional anesthesia particularly valuable.
To summarize, the Oxford Handbook of Anaesthesia offers an expansive volume of information in a clear, concise, easy-to-read manner. The text covers key concepts without getting too involved in the details, making it an excellent choice as a first text for medical students or junior residents interested in the specialty. I recommend this highyield book for any learner who wants to build a foundation of knowledge in anesthesiology.

Conflict of interest None declared.

Editorial responsibility This submission was handled by Dr. Hilary P. Grocott, Editor-in-Chief, Canadian Journal of Anesthesia. 